\title{
Problèmes de traduction posés par la siglaison dans le domaine des nouvelles technologies de l'information et de la communication
}

\section{Claudia Wolosin}

\section{OpenEdition}

\section{Journals}

Édition électronique

URL : http://journals.openedition.org/asp/3468

DOI : 10.4000/asp.3468

ISSN : 2108-6354

Éditeur

Groupe d'étude et de recherche en anglais de spécialité

\section{Édition imprimée}

Date de publication : 1 décembre 1996

Pagination : 147-160

ISSN : 1246-8185

\section{Référence électronique}

Claudia Wolosin, «Problèmes de traduction posés par la siglaison dans le domaine des nouvelles technologies de l'information et de la communication », ASp [En ligne], 11-14 | 1996, mis en ligne le 05 mai 2013, consulté le 19 avril 2019. URL : http://journals.openedition.org/asp/3468 ; DOI : 10.4000/ asp.3468

Ce document a été généré automatiquement le 19 avril 2019.

Tous droits réservés 


\title{
Problèmes de traduction posés par la siglaison dans le domaine des nouvelles technologies de l'information et de la communication
}

\author{
Claudia Wolosin
}

\section{Introduction}

1 La siglaison est un processus de réduction morphologique par lequel une lexie - ou groupe lexical - est réduite aux initiales ou aux éléments initiaux des mots qui le composent. Elle est très présente dans la création lexicale en langue contemporaine, en grande partie grâce au développement, à la démocratisation et à la vulgarisation des sciences et de la technologie où se multiplient les organismes et les concepts de toutes sortes. Cette matrice lexicogénique est particulièrement productive dans le domaine des nouvelles technologies de l'information et de la communication, désigné par le sigle NTIC (nouvelles technologies de l'information et de la communication).

2 L'instabilité et l'évolution rapide de la terminologie dans ce domaine posent de nombreux problèmes aux traducteurs d'ouvrages techniques. Cette étude sera consacrée à l'examen des processus d'identification et de traduction des sigles. L'anglais y sera désigné comme la langue source, et le français comme la langue cible.

3 Avant d'aborder ces différents problèmes, examinons rapidement deux phénomènes qui contribuent à la complexité de la traduction des documents des NTIC: l'instabilité terminologique, et les facteurs linguistiques et extra-linguistiques responsables de l'importante production de sigles dans ce domaine. 


\subsection{L'instabilité terminologique dans le domaine des NTIC}

\subsubsection{La lexicalisation dans la langue cible du terme provenant de la langue source}

4 L'évolution rapide des nouvelles technologies et leur caractère international contribuent à l'instabilité terminologique. Avant que la traduction officielle d'un terme ne soit établie dans la langue cible, il arrive souvent que le terme de la langue source soit déjà lexicalisé et bien ancré dans la langue cible, et que les usagers s'en accommodent très bien. Par voie de conséquence, les traductions de ces termes s'intègrent difficilement dans le lexique de la langue cible. Les terminologues se trouvent ainsi à la traîne en raison de la démocratisation technologique et, par conséquent, linguistique, de ce domaine. De plus, les traductions officielles de ces termes sont parfois méconnaissables, du moins au premier abord, aux francophones.

\subsubsection{La pluralité des termes}

Les traducteurs dans ce domaine se trouvent ainsi confrontés à une terminologie peu homogène et relativement disparate. Il arrive relativement souvent que, pour un seul terme en anglais, plusieurs termes existent en français. On peut citer l'exemple de browser , terme en anglais pour lequel on trouve au moins trois termes en français :

browser (dans sa version originale par commodité et par souci de se faire

comprendre) ;

logiciel de navigation (le terme français couramment accepté) ;

butineur (la traduction recommandée du terme logiciel de navigation).

\subsubsection{Considérations sociolinguistiques}

6 Parfois les sujets parlants de la langue cible rejettent la traduction d'un terme pour des motifs de préférence du terme d'origine. Ce phénomène est principalement lié à deux considérations sociolinguistiques distinctes mais complémentaires.

La cohérence communicative

7 La plupart des termes de la langue source préservés dans la langue cible - qu'il s'agisse de néologismes morphologiques, sémantiques ou morpho-sémantiques -, satisfont à un besoin de nommer, d'étiqueter un objet, concept ou processus. À la différence de la cryptologie argotique, l'usage maintenu des termes d'origine dans la langue cible se fait dans un souci de se faire comprendre. L'intention de la signification dans la terminologie technico-scientifique est placée à la fois du côté de l'émetteur et du récepteur. Cette réciprocité fondamentale à l'intérieur d'une communauté est assurée par un langage adopté dans la perspective de l'Autre. Ainsi la terminologie donne-t-elle une cohérence communicative au langage.

\section{L'appartenance}

8 Si une personne est connue par ses fréquentations, elle l'est aussi par la terminologie qu'elle emploie. Une terminologie n'est pas seulement un outil de communication, mais aussi un mécanisme d'inclusion et d'exclusion. La célèbre hypothèse de Whorf et Sapir, aujourd'hui connue de tous, avance que tout langage reflète fidèlement la façon de penser et de sentir de ceux qui le parlent, et manifeste non seulement le savoir collectif d'une communauté, mais également et surtout ses structures culturelles, sociales, religieuses et politiques. Bien que les débats centrés sur cette hypothèse aient été très animés et aient 
pu provoquer de nombreuses polémiques, il n'en reste pas moins qu'il existe réellement une liaison entre le langage d'une communauté et cette communauté, et ce lien est senti plus ou moins vivement par chacun de ses membres.

Inversement, toute communauté qui souhaite s'isoler d'une autre communauté linguistique plus vaste tend à développer son propre langage ou, dans le domaine des NTIC, sa propre terminologie. L'emploi de tel ou tel terme devient ainsi une sorte de signe distinctif d'appartenance, pouvant séparer des spécialistes des non-spécialistes, des «branchés » des « non-branchés », etc.

Dans le domaine des NTIC, on trouve de nombreux exemples de ce phénomène. Contentons-nous ici de citer l'exemple du terme shareware, qui, en dépit des traductions proposées (partagiciel, distributiel et contributiel), résiste à toute tentative d'évincement.

\subsubsection{Les usagers}

11 Enfin, le dernier facteur qui contribue à l'instabilité terminologique provient de la lexicalisation dans la langue cible de termes de la langue source. Cette lexicalisation donne lieu à de nombreux exemples de phraséologie où l'anglais et le français s'entremêlent. Ainsi trouve-t-on en français les expressions comme fichiers batch, faire $d u$ morphing, vendu en bundle ou acheter du shareware. Comme nous le verrons, ce problème est aggravé lorsqu'on a affaire à des sigles, en raison de leur caractère plus opaque par rapport à d'autres termes du lexique.

\subsection{Facteurs liés à la productivité de la siglaison dans le domaine des NTIC}

\subsubsection{Facteurs linguistiques}

\section{L'économie linguistique}

12 Il s'agit de la loi du moindre effort, promue par la réduction morphologique des termes techniques :

\begin{tabular}{|l|l|}
\hline ADPCM & Adaptive Differential Pulse Code Modulation \\
\hline MUSICAM & Masking pattern adapted Universal Sub-band Integrated Coding and Multiplexing \\
\hline
\end{tabular}

\section{L'opacité du terme}

13 Du fait de leur aspect compact, les sigles désignant des concepts ou des techniques parfois très complexes acquièrent ainsi une valeur iconique. De plus, il n'est pas toujours facile de discerner un sigle en tant que tel.

PAL, SECAM, Power PC, Modem, Antiope...

\subsubsection{Facteurs extra-linguistiques}

\section{Le support écrit}

Les facteurs extra-linguistiques sont en rapport étroit avec le support écrit. La plupart des communications entre utilisateurs des NTIC ont lieu par écrit, sur un support papier 
ou électronique. La siglaison est mieux adaptée aux modes de communication écrits qu'oraux.

\section{L'économie rédactionnelle et de l'espace sur le support}

L'écriture étant le mode de communication prépondérant dans ce domaine, le cryptage des termes techniques (et parfois non techniques) a l'avantage d'économiser du temps à la rédaction et à la lecture de textes ou de messages. Une fois de plus, il s'agit surtout de la loi du moindre effort.

Les sigles sont des formes graphiques compactes et représentent donc des gains d'espace linéaire sur le support. Comparons :

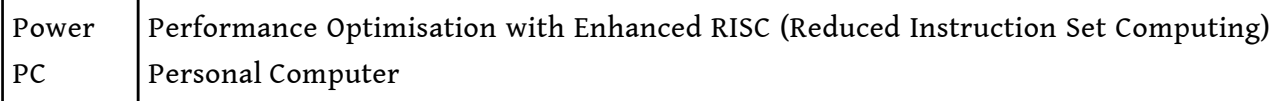

17 Les utilisateurs peuvent ainsi faire des économies d'énergie considérables grâce aux sigles. À titre d'exemple, comparons les deux énoncés suivants :

The NPS automatically handles TCP/IP, and interfaces with BSD, AIX, IBM MCS, MSDOS, and Mac OS.

The Network Processing System automatically handles Transmission Control Protocol/ Internet Protocol, and interfaces with Berkeley System Development, Advanced Interactive Executive, International Business Machines' Micro Channel System, Microsoft's Disk Operating System, and Macintosh's Operating System.

Ces énoncés comportent tous deux le même contenu sémantique. Toutefois, l'économie rédactionnelle du premier saute aux yeux : 81 caractères, contre 273 pour le second. La rédaction du premier énoncé représente alors une économie de l'ordre de $70 \%$.

En contrepartie, cette économie linguistique se règle en coût mémoriel. Parfois les signifiants réduits peuvent rendre la compréhension des textes très difficile, non seulement à cause de l'opacité des termes, mais aussi en raison de la complexité des concepts qu'ils véhiculent et de l'ambiguïté éventuelle du signifiant plein. De plus, l'auteur d'un texte comprenant un sigle relativement nouveau prendra la peine de fournir le signifiant plein ; mais, une fois que le sigle est plus ou moins intégré dans la communauté linguistique, son signifiant plein n'apparaît que très rarement dans les textes. Comment se souvenir de tous les signifiants pleins? Pire encore, comment trouver le signifiant plein d'un sigle lorsque ce premier a déjà disparu des textes techniques ? Les utilisateurs des NTIC ne restent pas toujours indifférents aux phénomènes linguistiques qui se produisent dans ce domaine, et expriment parfois leur frustration devant le nombre croissant de sigles, et ce, en terme de sigles. Par exemple, PCMCIA signifie Personal Computer Memory Card International Association, mais il signifie aussi People Can't Memorize Computer Instruction Acronyms! Nous pourrions également citer YABA : Yet Another Bloody Acronym!

\section{La siglaison}

\subsection{Définition}

La siglaison est l'un des trois processus de réduction morphologique ${ }^{1}$ qui donnent lieu à des néologismes. Elle consiste précisément en la réduction d'une lexie complexe aux 
initiales ou aux éléments initiaux des mots qui la composent. La siglaison est l'une des treize matrices lexicogéniques mises en œuvre dans la création lexicale anglaise.

Dans la très grande majorité des cas, la lexie réduite est un groupe nominal. Il semblerait que ces groupes nominaux s'intègrent rapidement dans le lexique de la langue du fait qu'ils sont dotés d'un statut de nom propre. Ainsi, même lorsque le signifiant plein correspondant au sigle est traduit, le sigle lui-même résiste souvent à la traduction.

Cette observation nous conduit à constater la caractéristique quasi iconique de certains sigles par rapport au sens qu'ils véhiculent. Pour cela, le sigle est souvent préservé dans sa forme graphique d'origine même après la traduction de son signifiant plein. La correspondance entre le terme d'origine et le terme traduit est alors garantie par leur signifiant commun.

Sur les 1341 entrées qui composent notre corpus, la grande majorité est constituée de substantifs. Cependant, on rencontre aussi quelques rares phrases simples :

\begin{tabular}{|l|l|}
\hline WYSIWYG & What You See Is What You Get \\
\hline YABA & Yet Another Bloody Acronym \\
\hline
\end{tabular}

et quelques adjectifs :

\begin{tabular}{|l|l|} 
Fichiers BMP & Fichiers BITMAP \\
\hline
\end{tabular}

\subsection{Sigles, acronymes et abréviations} point. Les lettres d'un sigle peuvent l'être aussi, mais on remarque de moins en moins l'usage de points dans les sigles. Le point semble être une marque de ponctuation réservée à l'abréviation, tandis que le sigle se veut plus opaque et lexicalisé. C.C. comme centre commercial). À la différence des abréviations, un sigle est lu en épelant lettre par lettre $(C-P-U)$ ou, lorsqu'il correspond à un modèle morpho-phonique, comme un mot normal (RAM). correspond à un modèle morpho-phonique et est lue comme un $\operatorname{mot}^{2}$. Lorsqu'à l'intégration phonique s'ajoute l'intégration graphique, c'est-à-dire l'emploi des lettres minuscules, l'acronyme est alors assimilé dans la langue en tant que mot à part entière ${ }^{3}$. (Tournier 1985 : 298) 
réduction peut se faire par une variété d'opérations réductives. Dans la langue anglaise, la siglaison se manifeste de façon fort variée. Jean Tournier a recensé trente-trois modèles de réduction morphologique sur lesquels sont formés les sigles et les acronymes (1985: 309-311). Bien sûr, ces modèles peuvent parfois se recouper partiellement et peuvent aussi se combiner entre eux. À titre d'exemple, voici cinq opérations réductives que l'on retrouve souvent dans les NTIC :

La réduction d'un groupe à l'initiale de chaque mot du groupe :

CPU : Central Processing Unit

La réduction d'un composé à une partie des syllabes :

modem : Modulator/demodulator

La création d'une forme correspondante à un modèle morpho-phonique à partir de divers éléments du groupe :

Cobol: Common Business-Oriented Language

La composition dont le premier élément est l'une des initiales du second :

Cassette DAT : Digital Audio Tape

L'ajout d'éléments vocaliques extérieurs à un sigle :

SCSI : scuzzy

Les sigles et les acronymes se forment donc grâce aux mêmes processus lexicogéniques. Pour cette raison, dans le cadre de cette étude, nous allons réunir sous le terme siglaison toute réduction d'éléments à leur partie initiale.

\subsection{Cas de siglaison dans la traduction}

31 Les problèmes posés par la traduction des sigles et de leur(s) signifiant(s) sont nombreux et variés. Les sigles constituent une terminologie complexe en raison du dédoublement du signifiant d'un même signifié. Autrement dit, chaque signifiant dans sa forme graphique et/ou phonologique pleine (que nous désignerons par S1), est également représenté par une forme graphique et phonologique plus compacte - le sigle -, que nous désignerons comme S2.

Une traduction réussie du S1 et du S2 provoque un effet exponentiel des termes, en créant deux autres signifiants pour un même signifié. Ce signifié sera alors désigné par deux signifiants dans la langue source et deux autres dans la langue cible, pour un total de quatre signifiants.

Les signifiants impliqués dans la traduction d'un sigle

\begin{tabular}{|l|l|l|}
\hline Signifiant & Description & Exemple \\
\hline S1 & Le signifiant complet de la langue source & Hard disk \\
\hline S2 & Le sigle de la langue source & HD \\
\hline S3 & La traduction du S1 dans la langue cible & Disque dur \\
\hline S4 & La traduction du S2 dans la langue cible & DD \\
\hline
\end{tabular}




\section{Le corpus} traduction du S4 n'est pas adoptée par les usagers de la langue cible. Ils maintiennent l'emploi du S2 malgré la traduction du S4 :

\begin{tabular}{|l|l|}
\hline S1 & Disk Operating System \\
\hline S2 & DOS \\
\hline S3 & Système d'exploitation de disquettes \\
\hline S4 & *SED \\
\hline
\end{tabular}

Dans huit dictionnaires spécialisés (dont trois bilingues) et plusieurs ouvrages en langue française consacrés aux NTIC, nous avons relevé 1341 sigles couramment employés dans ce domaine.

Des 1341 sigles recensés, nous avons identifié et écarté de cette étude ceux qui représentent des noms propres (noms d'organismes, d'instituts, de salons professionnels ou d'entreprises), soit un total de 113 (dont 36 en français et 76 en anglais). Restent donc 1228 sigles, dont 106 en français (soit $8 \%$ ) et 1123 en anglais (soit $92 \%$ ).

En examinant les 106 sigles en français, plusieurs observations ont été faites :

16 sigles dans la langue cible - soit $15 \%$ - sont identiques à leur équivalent dans la langue source. Autrement dit, le S1 est traduit en S3, et le S4 résultant est identique au S2:

\begin{tabular}{|l|l|}
\hline S1 & Audio-Visual \\
\hline S2 & AV \\
\hline S3 & Audio-Visuel \\
\hline S4 & AV \\
\hline
\end{tabular}

Parmi ces sigles, nous avons constaté que, dans $14 \%$ des cas - soit 15 sigles -, la

\section{Seulement 3 sigles en français n'ont pas d'équivalent en anglais (soit $2 \%$ ).}

Donc, des 106 sigles identifiés dans la langue française, seulement 72 -soit $67 \%$-sont réellement utilisés.

Sur la totalité du corpus (1 228), cela ne représente que $6 \%$ des sigles. Autrement dit, $94 \%$ des sigles identifiés dans les dictionnaires et les ouvrages francophones sont en anglais.

Revenons aux sigles ayant le même signifié et qui sont identiques dans les deux langues. Les S1 sont effectivement traduits en S3 (audiovisuel, infrarouge...). Mais pouvons-nous réellement considérer comme des traductions les S4 identiques aux S2 (AV, IR...) ? Ne serait-il pas plus juste de les considérer comme des S2 maintenus dans la langue cible, dans le souci de préserver et d'uniformiser la terminologie ? Cela signifierait alors que ces 
sigles identiques ne devraient pas compter parmi les sigles (S2) traduits. Dans ce cas, le nombre total de $\mathrm{S} 4$ traduits ne représentent que $5 \%$ du corpus.

Tournons-nous maintenant vers un examen de la démarche du traducteur face aux différents problèmes posés par ces cas d'espèce.

\section{La démarche du traducteur}

Le manque de systémicité et d'uniformisation de la terminologie complique énormément le travail du traducteur. Sa tâche principale consiste avant tout à rester vigilant et à savoir quels sont les termes traduits, non traduits, traduisibles, et enfin intraduisibles.

La démarche du traducteur se déroule en deux temps. Il doit d'abord procéder à l'identification et à la vérification de la définition d'un sigle. Ensuite, il doit rechercher une traduction existante éventuelle, puis prendre la décision d'adopter ou non le sigle traduit.

Nous avons identifié quatre cas d'espèce liés à l'identification et à la définition d'un terme, et sept problèmes liés à la traduction.

\subsection{Problèmes d'identification et de définition}

Un sigle dans la langue source est homonymique. S2 représente alors S1, S1', S1" ou S1"'. L'identification consiste alors à trouver le S1 correspondant en fonction du contexte.

\begin{tabular}{|l|l|}
\hline CAT (S2) & $\begin{array}{l}\text { Compact Audio Technology (S1) } \\
\text { Computer Axial Tomography (S1) } \\
\text { Computer-Assisted Teaching (S1) }\end{array}$ \\
\hline DSS(S2) & $\begin{array}{l}\text { Decision Support System (S1) } \\
\text { Digital Signal Standard (S1) } \\
\text { Distributed Security Service (S1) }\end{array}$ \\
\hline
\end{tabular}

Un même sigle existe dans la langue source et dans la langue cible mais ne désigne pas les mêmes S1. L'identification doit permettre de distinguer les différents termes et de ne pas interpréter le S2 comme étant représentatif d'un terme commun aux deux langues.

\begin{tabular}{|l|l|}
\hline BD & $\begin{array}{l}\text { Base de données } \\
\text { Boot Descriptor }\end{array}$ \\
\hline CMS & $\begin{array}{l}\text { Composant monté en surface } \\
\text { Cable Management System }\end{array}$ \\
\hline RLE & $\begin{array}{l}\text { Réseau Local d'Entreprise } \\
\text { Run Length Encoding }\end{array}$ \\
\hline
\end{tabular}


Réseau Téléphonique Commuté

Return to Control

\section{L'incertitude des S1}

$\mathrm{Au}$ cours des recherches terminologiques, le traducteur trouve parfois deux ou plusieurs variations d'un même signifiant plein. Il se doit alors de vérifier l'exactitude du signifiant plein avant de l'intégrer dans la traduction. Comparons :

\begin{tabular}{|l|l|}
\hline COM (S2) & $\begin{array}{l}\text { Common Object Model (?) } \\
\text { Component Object Model (?) }\end{array}$ \\
\hline GDI(S2) & $\begin{array}{l}\text { Graphic Development Interface (?) } \\
\text { Graphics Driver Interface (?) }\end{array}$ \\
\hline PMMU(S2) & $\begin{array}{l}\text { Paged-Memory Management Unit (?) } \\
\text { Programmable Memory } \\
\text { Management Unit (?) }\end{array}$ \\
\hline
\end{tabular}

Plusieurs sigles se ressemblent graphiquement mais ne désignent pas les mêmes S1. Une fois de plus, une grande vigilance s'impose du fait que les rédacteurs d'ouvrages techniques négligent parfois l'exactitude typographique :

\begin{tabular}{|l|l|}
\hline MIP & Modulation d'Impulsions en Position \\
\hline MIPS & Music Information Processing Standards \\
\hline Mips & Millions of Instructions per Second \\
\hline
\end{tabular}

\subsection{Cas de traduction}

La traduction du S2 en S4 existe et est couramment employé dans la langue cible :

\begin{tabular}{|l|l|}
\hline S1 & DataBase Management System \\
\hline S2 & DBMS \\
\hline S3 & Système de Gestion de Bases de Données \\
\hline S4 & SGBD \\
\hline S1 & Error Correction Code \\
\hline S2 & ECC \\
\hline
\end{tabular}




\begin{tabular}{|l|l|}
\hline S3 & Code de Correction d'Erreurs \\
\hline S4 & CCE \\
\hline
\end{tabular}

51 Aucune traduction du S2 n'existe. La version anglaise est utilisée. Dans ce cas, le S1 est traduit en S3, mais les deux langues partagent le S2 :

\begin{tabular}{|l|l|}
\hline S1 & Compact Disc \\
\hline S2 & CD \\
\hline S3 & Disque compact \\
\hline S4 & CD \\
\hline
\end{tabular}

ou encore

\begin{tabular}{|l|l|}
\hline S1 & Random Access Memory \\
\hline S2 & RAM \\
\hline S3 & Mémoire vive \\
\hline S4 & RAM \\
\hline
\end{tabular}

52 La traduction du sigle existe, mais n'est rarement ou jamais utilisée. Dans notre corpus nous avons trouvé 15 exemples de ce type (sur 106 traductions, soit $14 \%$ ). Le traducteur doit tenir compte de la nature du document à traduire et des destinataires éventuels lors du choix du terme à employer.

53 En règle générale, plus l'ouvrage est vulgarisé, plus on a tendance à employer le terme (S2) de la langue source. N'oublions pas que, dans la grande majorité des cas, ces termes sont lexicalisés bien avant l'apparition d'une traduction. Comme nous l'avons dit plus haut, le S2 résiste souvent à la fois à la traduction (S4) et à l'évincement dans l'éventualité où une traduction officielle existe. L'emploi de la traduction officielle d'un sigle, une fois imposé, commence souvent par les fabricants français de produits informatiques et de logiciels. Mais en attendant que ces produits gagnent du terrain sur le marché, le S4 risque de n'être connu que par un nombre réduit de spécialistes dans le domaine (et encore faut-il que ces derniers l'emploient). Il y a donc de fortes chances pour que davantage de lecteurs reconnaissent le S2, et que l'emploi de ce dernier facilite la compréhension du texte.

Dans le cas contraire, si le traducteur opte pour le terme traduit (mais moins couramment employé et peut-être moins connu des lecteurs), il doit insérer une petite parenthèse précisant l'origine du S4, c'est-à-dire le S3, et éventuellement le S2 et le S1. 
Quelques S2 utilisés à la place des S4

\begin{tabular}{|l|l|l|l|}
\hline langue source & & langue cible & \\
\hline S1 & S2 & S3 & S4 \\
\hline Asynchronous Transfer Mode & ATM & Mode de Transfer Asynchrone & *MTA \\
\hline Pulse Code Modulation & PCM & Modulation par Impulsions en codage & *MIC \\
\hline Local Area Network & LAN & Réseau Local en Entreprise & *RLE \\
\hline
\end{tabular}

Les traductions ne sont pas systématiques. Le plus souvent, il s'agit d'une composition dont le premier élément est l'une des initiales du second. Encore une fois, une vigilance et un grand travail de vérification s'imposent. Le traducteur se voit souvent dans l'obligation d'ouvrir une parenthèse pour expliquer l'origine du terme. Observons le manque de systémicité dans les exemples suivants :

\begin{tabular}{|l|l|}
\hline S1 & Bit (Binary digit) \\
\hline S3 & élément binaire \\
\hline
\end{tabular}

mais

\begin{tabular}{|l|l|}
\hline S1 & Bits Per Inch \\
\hline S2 & BPI \\
\hline S3 & bits par pouce \\
\hline S4 & bpp \\
\hline
\end{tabular}

ou encore

\begin{tabular}{|l|l|}
\hline $\mathrm{S}_{1}$ & Messaging Application Programming \\
\hline S2 & MAPI \\
\hline S3 & Messagerie API \\
\hline S4 & MAPI \\
\hline
\end{tabular}

Parfois la traduction du signifiant plein (S1) est influencée par le souci de préserver le sigle (S2) dans la langue cible. Nous avons trouvé 16 sigles en français (soit $15 \%$ du corpus) qui s'insèrent dans cette catégorie, dont : 


\begin{tabular}{|l|l|}
\hline $\mathrm{S}_{1}$ & Electronic Data Interchange \\
\hline $\mathrm{S} 2$ & EDI \\
\hline S3 & Échange de Données Informatisées \\
\hline S4 & EDI \\
\hline
\end{tabular}

ou encore

\begin{tabular}{|l|l|}
\hline $\mathrm{S}_{1}$ & Multiplexed Analog Components \\
\hline S2 & MAC \\
\hline S3 & Multiplex Analogique en Composants \\
\hline S4 & MAC \\
\hline
\end{tabular}

57 Le phénomène de conversion est très courant en anglais, et les sigles n'y échappent pas. La conversion se produit lorsqu'un mot change de classe, par exemple en passant de la classe nominale à la classe verbale, ou inversement. La plupart du temps, un sigle change de classe de mots en passant de la classe nominale à la classe verbale. La traduction de ces nouveaux verbes se fera en tenant compte de la fonction technique décrite par le signifiant plein.

\begin{tabular}{|l|l|}
\hline FTP & envoyer par FTP \\
\hline MUD & participer dans un MUD \\
\hline BinHex & binhexer, débinexer, encoder/décoder en BinHex \\
\hline Telnet & telnetter, envoyer par Telnet \\
\hline
\end{tabular}

Parfois la conversion se produit de la classe nominale à la classe adjectivale :

to perform a Veronica search : effectuer une recherche avec Veronica

Certains sigles sont artificiels, c'est-à-dire qu'ils sont créés après la création d'un terme. Dans ces cas, le S1 devient un S2 sans subir aucune réduction morphologique. Les sigles artificiels témoignent souvent de l'élément ludique du langage. Pour cette raison, le traducteur doit tenir compte de la nature de l'ouvrage à traduire, afin de déterminer si oui ou non une parenthèse explicative mériterait d'y être incluse.

\begin{tabular}{|l|l|}
\hline Jughead & Jonzy's Universal Gopher Hierarchy Excavation \& Display \\
\hline Veronica & $\begin{array}{l}\text { Very Easy Rodent-Oriented Net-Index of Computerized Archives (Index d'archives } \\
\text { électroniques très simple et orienté rongeur) }\end{array}$ \\
\hline
\end{tabular}


Enfin, certains sigles non techniques employés dans le domaine témoignent de la motivation ludique du langage et de l'esprit ludique des «infonautes» et des « multimédiaques » :

\begin{tabular}{|l|l|}
\hline POTS & Plain Old Telephone System \\
\hline RTFM & Read The F... Manual! \\
\hline WYSIWYG & What You See Is What You Get \\
\hline WYSIAWYG & WYSI Almost WYG \\
\hline WYSIAYG & WYSI All YG \\
\hline
\end{tabular}

\section{La pluri-terminologie des NTIC}

60 La pluri-terminologie des NTIC complique énormément le choix d'une terminologie. Le devoir du traducteur consiste à connaître et à mettre en œuvre les terminologies employées dans le domaine des NTIC. Préalable à toute traduction, son travail consiste à définir les facteurs suivants.

61 Connaître la nature de l'ouvrage. Est-il de nature très spécialisée, contenant une terminologie très rigide, et éventuellement destinée à un public très ciblé ? (Par exemple dans le cas de la traduction d'un logiciel ou d'un manuel de l'utilisateur.) Ou s'agit-il d'un ouvrage vulgarisé, destiné au grand public, dans lequel la terminologie est moins stricte ou doit être simplifiée?

62 Connaître le public auquel l'ouvrage est destiné. Est-ce un public spécialisé, familiarisé avec les sigles que contient l'ouvrage ? Ou s'agit-il d'un public moins averti, auquel cas le traducteur doit-il se donner pour tâche d'apporter des précisions quant aux S3 (et éventuellement les $\mathrm{S} 1$ ) des sigles employés?

63 Connaître la terminologie du public. Quels sont les termes utilisés et/ou connus de tous? Quelle est la terminologie la plus couramment employée et acceptée ? (N’oublions pas que la cohésion communicative et l'appartenance sont deux facteurs à ne pas perdre de vue lors du choix d'une terminologie.)

64 Le traducteur doit également identifier les termes nouveaux éventuels introduits dans l'ouvrage et les expliquer. Identifier et éventuellement éliminer les sigles inutiles ou pouvant perturber les lecteurs (par exemple les sigles ludiques ou artificiels). Enfin, il faut assurer une cohérence terminologique de l'ensemble. Cela peut paraître une grande évidence. Mais, compte tenu de tous les cas d'espèce que nous venons de passer en revue, cette cohérence terminologique semble loin d'être acquise. 


\section{BIBLIOGRAPHIE}

Brandeis, P. et F. Leroy. 1992. Dictionnaire de l'informatique. Paris : Dunod.

DeVos, J-C. et M. 1992. Les mots de la micro. Paris : Dunod.

Delalande, Y. 1990. Dictionnaire bilingue d'informatique. Alleur, Belgique : Marabout.

Engst, A. 1995. Internet pour Windows. Paris : Simon \& Schuster Macmillan.

Herellier, J-M. et C. Wolosin. 1994. Petit dictionnaire du multimédia. Paris : Sybex.

Jemaa, F. 1995. Multimédia - 2500 mots pour comprendre. Paris : Eyrolles.

Odier, A. et M. Zennaki. 1992. Dictionnaire des télécommunications. Alleur, Belgique : Marabout.

Person, R. 1995. Excel 95. Paris : Simon \& Schuster Macmillan.

Raymond, E. 1989. The New Hacker's Dictionary. Cambridge, MA : MIT Press.

Stanek, W. et L. Purcell. 1996. L'édition électronique. Paris : Simon \& Schuster Macmillan.

Tournier, J. 1985. Introduction descriptive à la lexico-génétique de l'anglais contemporain. Genève : Éditions Slatkine.

\section{Bibliographie complémentaire}

BABEL. A glossary of Computer-related Abbreviations and Acronyms <http://www.cs.tut.fi/tlt/ stuff/misc/babel.html >.

Hypertext Handbook <http://world.std.com>.

\section{NOTES}

1. Les deux autres matrices lexicogéniques de réduction morphologique d'un signifiant sont les processus de troncation: l'aphérèse (ou la troncation antérieure) (exemple : le bus); l'apocope (ou la troncation postérieure) (exemple : la fac). La siglaison et la troncation donnent lieu à des néologismes morphologiques. Cependant, à la différence des sigles, la lexicalisation des formes tronquées est relativement restreinte. Ces formes apparaissent souvent dans le discours, mais ne s'intègrent que très rarement dans le lexique de la langue. En revanche, la siglaison semble plus productive dans la langue écrite et s'intègre alors plus facilement dans le lexique proprement dit. Pour une discussion détaillée sur ces matrices lexicogéniques, voir Tournier (1985 : 297-307).

2. Par exemple, PAL ou SECAM.

3. Par exemple, laser, radar, Power PC. 


\section{RÉSUMÉS}

La siglaison est très productive dans le domaine des nouvelles technologies de l'information et de la communication. Ce processus cryptique contribue à l'instabilité terminologique du domaine, et pose de nombreux problèmes d'identification et de traduction aux traducteurs d'ouvrages spécialisés. Cet article examine les différents cas d'espèce, et tente d'établir un certain nombre de critères permettant au traducteur d'adopter une terminologie appropriée par rapport à l'ouvrage à traduire.

Acronyms are very productive in the field of information technology. This form of encryption contributes to the terminological instability in this field, and creates several problems for technical translators insofar as they must choose between different terminologies. This article presents the different problems linked to identifying and translating acronyms, and suggests criteria allowing for the selection of an appropriate terminology with respect to the document being translated.

\section{INDEX}

Keywords : acronym, information technology, pluri-terminology, terminology

Mots-clés : NTIC, pluri-terminologie, sigle, terminologie

\section{AUTEUR}

\section{CLAUDIA WOLOSIN}

Claudia Wolosin est ATER à l'Université Stendhal Grenoble 3. Elle y prépare un doctorat sous la direction de M. François Chevillet. Elle fait partie du groupe de recherche en traduction spécialisée, le GREMUTS, que dirige Elisabeth Lavaut-Olléon. Elle est co-auteur d'un dictionnaire de termes multimédia et traductrice d'ouvrages techniques. 